\title{
INFLUENCE OF DOPING ON THE LATTICE DYNAMICS OF GALLIUM NITRIDE
}

\author{
A. Kaschner, H. Siegle*, A. Hoffmann, C. Thomsen \\ Institut für Festkörperphysik, Technische Universität Berlin, Hardenbergstraße 36, 10623 Berlin, \\ Germany \\ *Present address: Lawrence Berkeley National Laboratory, Berkeley, California 94720, USA \\ U. Birkle, S. Einfeldt, D. Hommel
}

Institut für Festkörperphysik, FB 1, Univ. Bremen, Kufsteiner Str. NW 1, 28359 Bremen, Germany

Cite this article as: MRS Internet J. Nitride Semicond. Res. 4S1, G3.57 (1999)

\begin{abstract}
$\underline{\text { Abstract }}$
We present results of Raman-scattering experiments on $\mathrm{GaN}$ doped with $\mathrm{Si}, \mathrm{C}$, and $\mathrm{Mg}$, respectively, grown by molecular beam epitaxy (MBE). The influence of the different dopants on strain and freecarrier concentration was investigated. Furthermore, we report on several local vibrational modes (LVM) around $2200 \mathrm{~cm}^{-1}$ in Raman spectra of highly Mg-doped GaN. A possible explanation of these high-energy modes in terms of hydrogen-related vibrations is given. We also found a variety of new structures in the range of the GaN host lattice phonons. Secondary ion mass spectroscopy (SIMS) was applied to determine the concentration of magnesium and unintentionally incorporated hydrogen.
\end{abstract}

\section{$\underline{\text { Introduction }}$}

Much attention has been paid to the wide-bandgap material GaN due to its high potential for optoelectronic and high-power electronic applications [1]. Controlled p- and n-doping is a major issue for the fabrication of electronic devices based on group III-Nitrides. The growth of doped GaN epilayers and heterostructures by molecular beam epitaxy offers the advantage of lower unintentional dopant concentration in the material. Among the optical properties of doped GaN the influence of doping on the lattice dynamics is of special interest. Raman-spectroscopy is a powerful tool to investigate the correlation between doping, strain [2], and free-carrier concentration [3].

Moreover, dopant atoms can give rise to local vibrational modes (LVM) due to their different masses compared with those of the substituted elements [4]. High-energy modes are reported for Mg-doped GaN [5,6] which were assumed to be related to hydrogen complexes. This is of special interest, because hydrogen is known as a compensating center for magnesium acceptors. To our knowledge, no LVM for $\mathrm{Si}-, \mathrm{C}-$, or $\mathrm{Mg}$-doped $\mathrm{GaN}$ in the range of the host lattice phonons have been reported in literature so far.

\section{$\underline{\text { Experiment }}$}

The Raman-scattering experiments were carried out in backscattering geometry with a triple-grating spectrometer equipped with a cooled charge-coupled device detector. The $488 \mathrm{~nm}$ line of an $\mathrm{Ar}^{+} / \mathrm{Kr}^{+}$ mixed-gas laser was used for excitation. The Raman shifts were determined with an accuracy better than $1 \mathrm{~cm}^{-1}$. Microscope optics allowed spatially-resolved measurements with a resolution of about $0.7 \mu \mathrm{m}$. 
The samples under study were $\mathrm{Si}$-, $\mathrm{C}$-, and $\mathrm{Mg}$-doped $\mathrm{GaN}$ films of about $1 \mu \mathrm{m}$ thickness grown on sapphire (0001) substrates by MBE; the details are given elsewhere [7]. The Mg-doped samples A and $\mathrm{B}$ were p-conductive with a hole concentration at room temperature of $3.7 \cdot 10^{17} \mathrm{~cm}^{-3}$ and $1.4 \cdot 10^{17} \mathrm{~cm}^{-3}$, respectively, whereas the other two samples were compensated but n-conductive. Secondary ion mass spectroscopy was applied to determine the concentration of magnesium and hydrogen. The measured concentrations are accurate to within $20 \%$ deviation. The dopant concentration of the $\mathrm{Si}$ - and $\mathrm{C}$-doped $\mathrm{GaN}$ films were estimated in terms of growth conditions. The free-electron concentration in the Si-doped samples varied from $8.1 \cdot 10^{15} \mathrm{~cm}^{-3}$ to $1.3 \cdot 10^{20} \mathrm{~cm}^{-3}$ as determined by Hall measurements.

\section{$\underline{\text { Results }}$}

\section{Local vibrational modes in doped GaN}

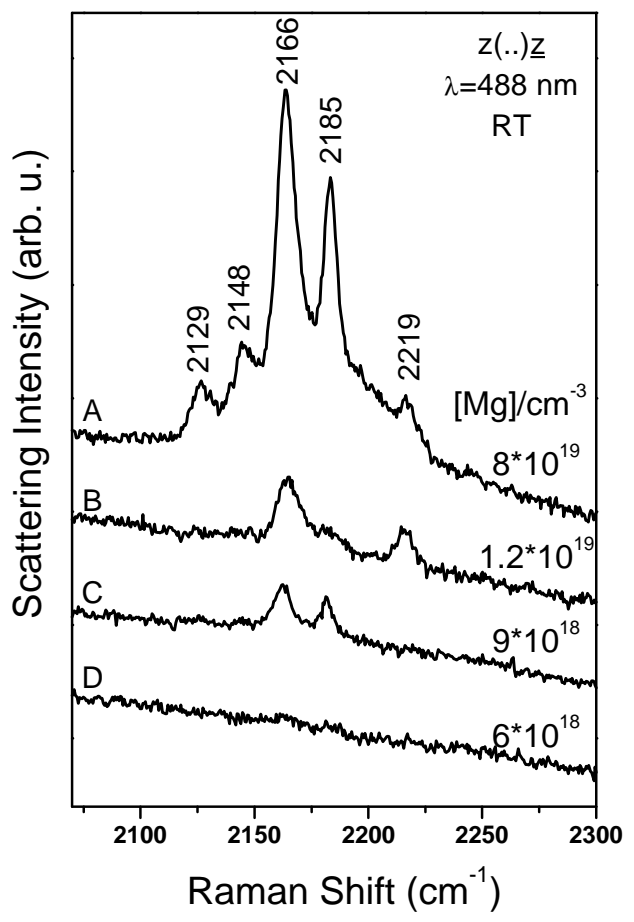

Figure 1: Room-temperature Raman spectra of GaN with different magnesium content in the high-energy range.
Figure 1 shows Raman spectra of samples with the respective magnesium concentrations of $8 \cdot 10^{19} \mathrm{~cm}^{-3}(\mathrm{~A})$, $1.2 \cdot 10^{19} \mathrm{~cm}^{-3}(\mathrm{~B}), 9 \cdot 10^{18} \mathrm{~cm}^{-3}(\mathrm{C})$ and $6 \cdot 10^{18} \mathrm{~cm}^{-3}(\mathrm{D})$ in the high-energy region. In the spectrum of sample A with the highest magnesium concentration a new mode appears at $2129 \mathrm{~cm}^{-1}$ in addition to the four LVM described in Ref. 5 . Apparently, the intensity of the modes correlates with the magnesium content. A $\mathrm{Mg}$-concentration of around $1 \cdot 10^{19} \mathrm{~cm}^{-3}$ is necessary for some of the high-energy modes to appear well-resolved in the spectra. The hydrogen concentration for all the samples investigated is about $1 \cdot 10^{19} \mathrm{~cm}^{-3}$ as determined by secondary ion mass spectroscopy. Since hydrogen was not intentionally supplied during growth its incorporation possibly arises from the residual water vapor pressure in the growth chamber.

Yi et al. [8] observed a similar structure of five modes around $2800 \mathrm{~cm}^{-1}$ in $\mathrm{Mg}$-doped $\mathrm{GaN}$ grown by metalorganic vapor phase epitaxy (MOVPE). These modes were attributed to symmetric and asymmetric $\mathrm{C}-\mathrm{H}_{\mathrm{n}}$ $(n=1,2,3)$ vibrations due to carbon incorporation caused by the decomposition of the magnesium precursor during growth. Remarkably, the frequency ratio for all the five modes is between 1.33 and 1.34. This is close to $\sqrt{2}$, a value expected for similar vibrations with carbon $\left(\mathrm{m}_{\mathrm{C}}=12.01 \mathrm{u}\right)$ replaced by magnesium $\left(\mathrm{m}_{\mathrm{Mg}}=24.31 \mathrm{u}\right)$. This implies that magnesium is built into the lattice on a nitrogen site with force constants similar to those of carbon, an interpretation which seems doubtful. Furthermore, there are experimental and theoretical articles that determined the frequency of the $\mathrm{Mg}-\mathrm{H}$ vibration above $3000 \mathrm{~cm}^{-1}[5,9]$.

Alternatively, hydrogen-decorated native defects or hydrogen at extended defects such as dislocations can give rise to high-energetic vibrations. When the Fermi level reaches a value below $2.1 \mathrm{eV}$ the formation of $\mathrm{V}_{\mathrm{Ga}}-\mathrm{H}_{\mathrm{n}}$ complexes with $\mathrm{n}=1,2,3$ is likely to occur with a predicted frequency of $3100 \mathrm{~cm}^{-1}$ for a $\mathrm{V}_{\mathrm{Ga}}-\mathrm{H}$ complex [10]. This is much higher than the frequencies we observe, even if anharmonic terms may lower the frequency considerably [10]. 
However, we rather believe that the magnesium incorporation causes the high-energy modes indirectly by creating defects or by lowering the Fermi level and therefore making the the hydrogen

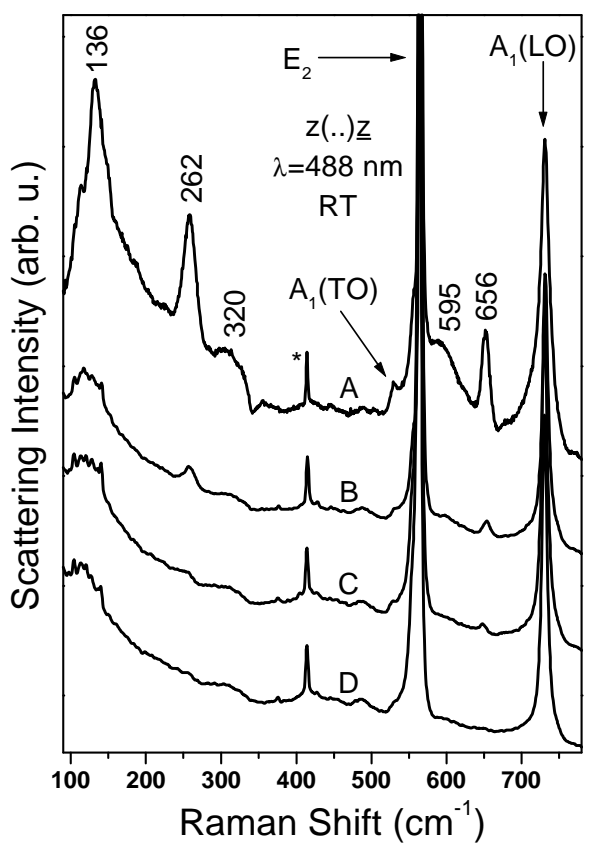

Figure 2: Room-temperature Raman spectra (normalized to $\mathrm{E}_{2}$ intensity) of $\mathrm{GaN}$ with different magnesium content in the lowenergy range. Apart from the host lattice phonons five additional modes are observed. The peak marked by an asterisk is a phonon from the sapphire substrate. complex formation likely [11].

Apart from the five high-energy modes the highly $\mathrm{Mg}$ doped samples exhibit new vibrational modes in the region of the acoustic and optical $\mathrm{GaN}$ phonons. We found structures at $136 \mathrm{~cm}^{-1}, 262 \mathrm{~cm}^{-1}, 320 \mathrm{~cm}^{-1}, 595 \mathrm{~cm}^{-1}$ and $656 \mathrm{~cm}^{-1}$. Figure 2 displays the low-energy part of the Raman spectra of the four samples in the same sequence as in Figure 1. The broad structures centered around $320 \mathrm{~cm}^{-1}$ and $595 \mathrm{~cm}^{-1}$ may result from disorder-activated scattering, in which built-in defects yield a relaxation of the $\mathrm{q}=0$ selection rule for first-order Raman scattering. This interpretation is supported by the fact that from sample $D$ to $A$ the forbidden $A_{1}$ (TO) mode at $533 \mathrm{~cm}^{-1}$ increases considerably in intensity. Limmer et al. [12] reported on a disorder-activated Raman-mode around $300 \mathrm{~cm}^{-1}$ in ion-implanted $\mathrm{GaN}$ which is close to our $320 \mathrm{~cm}^{-1}$ mode. Furthermore, the cut-off at $340 \mathrm{~cm}^{-1}$ fits well with calculated phonon dispersion curves [13, 14]. On the other hand, the modes at $136 \mathrm{~cm}^{-1}, 262 \mathrm{~cm}^{-1}$ and $656 \mathrm{~cm}^{-1}$ probably do not originate from disorderactivated scattering since the phonon density of states (PDOS) does not exhibit marked structures in this frequency range [14]. Recently, a mode at $656 \mathrm{~cm}^{-1}$ was observed in GaN after annealing at $1000^{\circ} \mathrm{C}$ and related to a damaged-sapphire substrate [15]. The mode we observed at $656 \mathrm{~cm}^{-1}$ is of different origin since it does not scale with the intensity of the sapphire mode at $418 \mathrm{~cm}^{-1}$ nor with other modes of the damaged-sapphire at e.g. $770 \mathrm{~cm}^{-1}$ but rather with the Mg-concentration.

We thus believe these structures in the low-energy range to be correlated with magnesium. Equation (1) gives us a rough estimate of the magnesium local vibrational mode frequency from the effective masses of the GaN and LVM vibrations.

$$
\frac{\omega_{\mathrm{GaN}}}{\omega_{\mathrm{LVM}}} \approx \sqrt{\frac{\mu_{\mathrm{LVM}}}{\mu_{\mathrm{GaN}}}}
$$

Assuming that magnesium occupies a gallium site we obtain a value of about $640 \mathrm{~cm}^{-1}$ for N-Mg vibrations using $\omega_{\mathrm{GaN}}=\omega\left(\mathrm{E}_{1}(\mathrm{TO})\right)=560 \mathrm{~cm}^{-1}$.

Though the $656 \mathrm{~cm}^{-1}$ mode lies in the range of the optical phonons its observation may be possible because the PDOS is relatively low in the region from $640 \mathrm{~cm}^{-1}$ to $675 \mathrm{~cm}^{-1}$ as confirmed by secondorder Raman-scattering [13] and time-of-flight neutron spectroscopy [14]. We hence assign the $656 \mathrm{~cm}^{-1}$ mode to a local vibrational mode of magnesium in GaN. The $\mathrm{Mg}$-concentration in the $10^{19} \mathrm{~cm}^{-3}$ range is apparently sufficient to observe the LVM without resonant excitation.

The nature of the $136 \mathrm{~cm}^{-1}$ and $262 \mathrm{~cm}^{-1}$ mode remains unclear. Since the density of states is also relatively low in the energy range of the two modes in question it is conceivable that they are also LVM.

We did not observe any LVM for the $\mathrm{Si}$ - and $\mathrm{C}$-doped $\mathrm{GaN}$ samples in low-energy range nor in the high-energy range. 


\section{Biaxial Stress due to incorporation of dopants}

Due to its nonpolar character the $\mathrm{E}_{2}$ phonon frequency is a good measure for biaxial stress in $\mathrm{GaN}$ layers. Biaxial compressive stress increases the phonon frequency. Values of $4.2 \mathrm{~cm}^{-1} / \mathrm{GPa}$ [2], $6.2 \mathrm{~cm}^{-1} / \mathrm{GPa}$ [16] and $7.7 \mathrm{~cm}^{-1} / \mathrm{GPa}$ [17] have been reported. Figure 3 shows the dependence of the $\mathrm{E}_{2}$ frequency on the dopant concentration. While the $\mathrm{E}_{2}$ values remain nearly constant between $567 \mathrm{~cm}^{-1}$ and $568 \mathrm{~cm}^{-1}$ over the whole doping range in case of the silicon doping series, we see a strong hardening in the magnesium series for Mg-concentrations exceeding $1 \cdot 10^{19} \mathrm{~cm}^{-3}$. This

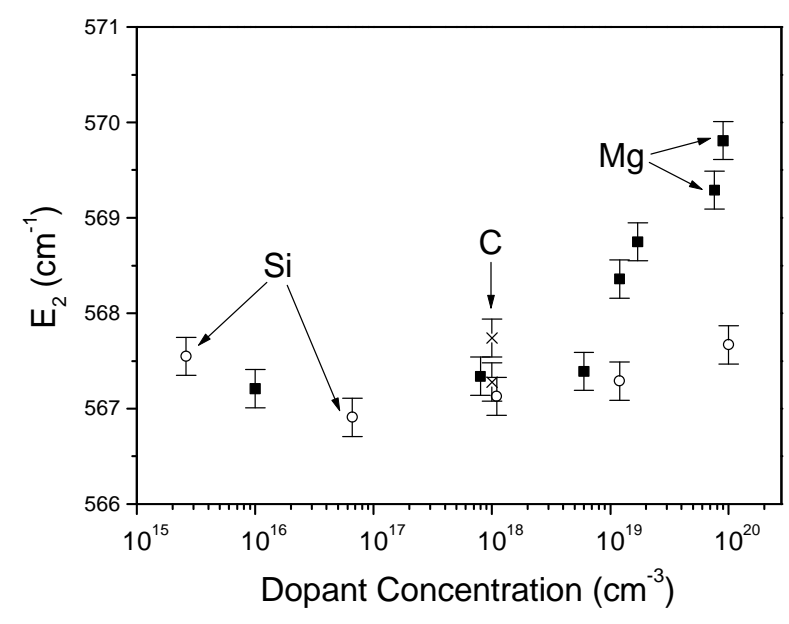

Figure 3: Position of the $E_{2}$ mode for different doped GaN samples.

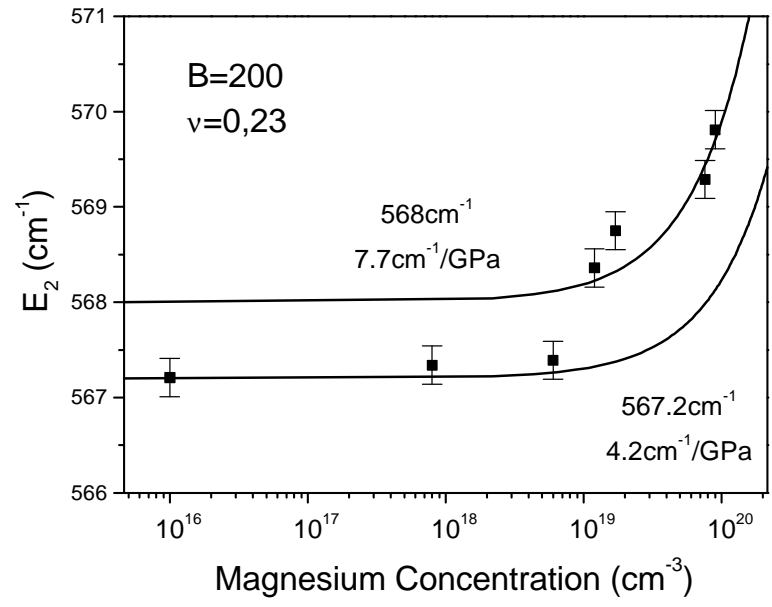

Figure 4: $E_{2}$ position vs. Mg-concentration. The lines show the predicted behavior using eq. (2) and (3) with different values for the bulk $E_{2}$ frequency and the $E_{2}$ shift. The values for $B$ and $v$ are taken from Ref. 2.

behavior can be explained by the different size of the dopant atom $\left(\mathrm{r}_{\mathrm{Mg}}=0.14 \mathrm{~nm}\right)$ and the replaced host atom $\left(\mathrm{r}_{\mathrm{Ga}}=0.126 \mathrm{~nm}\right)$. Using equation (2) one can calculate the influence of this effect on the strain [2].

$$
\varepsilon=\frac{\mathrm{c}-\mathrm{c}_{0}}{\mathrm{c}_{0}}=\frac{\left[1-\left(\frac{\mathrm{r}_{\mathrm{Mg}}}{\mathrm{r}_{\mathrm{Ga}}}\right)^{3}\right] \cdot \mathrm{C}}{3 \mathrm{~N}}
$$

Here, $\mathrm{N}$ is the number of lattice sites of the host matrix, $\mathrm{C}$ the dopant concentration, $\mathrm{c}$ and $\mathrm{c}_{0}$ the strained and unstrained lattice constant, respectively. Equation (3), where B is the bulk modulus and $v$ is the Poisson ratio, correlates the strain with the biaxial stress.

$$
\sigma=\varepsilon \cdot \frac{B}{v}
$$

Figure 4 shows the experimental data of the Mg-doped samples and calculated curves with different values for the strain-free $\mathrm{E}_{2}$ frequency and $\Delta \omega\left(\mathrm{E}_{2}\right) / \Delta \sigma$. It is obvious that the observed behavior can be explained by a size effect qualitatively, but the uncertainty for the basic GaN material parameters renders exact calculations difficult.

When substituting gallium by silicon one would expect the same effect but with opposite sign since the atomic radius of silicon is smaller than that of gallium. Surprisingly, we did not observe any significant shift for the $\mathrm{E}_{2}$ frequency up to a silicon concentration of $1 \cdot 10^{20} \mathrm{~cm}^{-3}$. The highly Si-doped samples do also exhibit a large free-carrier concentration (see next chapter). Therefore we believe, 
that the size effect is compensated by the change of the lattice constant due to free electrons [17]. Following Ref. 18 a free-carrier concentration of $5 \cdot 10^{19} \mathrm{~cm}^{-3}$ should increase the lattice constant by $0.01 \%$ which equals the absolute value of the size effect caused by the same silicon concentration.

The carbon concentration of the samples in our study is too low to observe a significant $\mathrm{E}_{2}$ shift.

\section{Determination of the free-carrier concentration}

The interaction of free charge carriers with longitudinal optical phonons leads to the observation of longitudinal phonon plasmon modes (LPP) [3]. If damping can be neglected the frequency of the LPP modes is given by equation (4).

$$
\left(\omega_{\mathrm{LPP}}^{ \pm}\right)^{2}=0.5 \cdot\left\{\omega_{\mathrm{L}}^{2}+\omega_{\mathrm{P}}^{2} \pm\left[\left(\omega_{\mathrm{L}}^{2}+\omega_{\mathrm{P}}^{2}\right)^{2}-4 \omega_{\mathrm{P}}^{2} \omega_{\mathrm{T}}^{2}\right]^{\frac{1}{2}}\right\}
$$

Here, $\omega_{\mathrm{L}}$ and $\omega_{\mathrm{T}}$ are the frequencies of the LO and TO phonons, respectively. The plasmon frequency is correlated with the free-carrier concentration.

$$
\omega_{\mathrm{P}}^{2}=\frac{\mathrm{ne}^{2}}{\mathrm{~m}^{*} \varepsilon_{\infty} \varepsilon_{0}}
$$

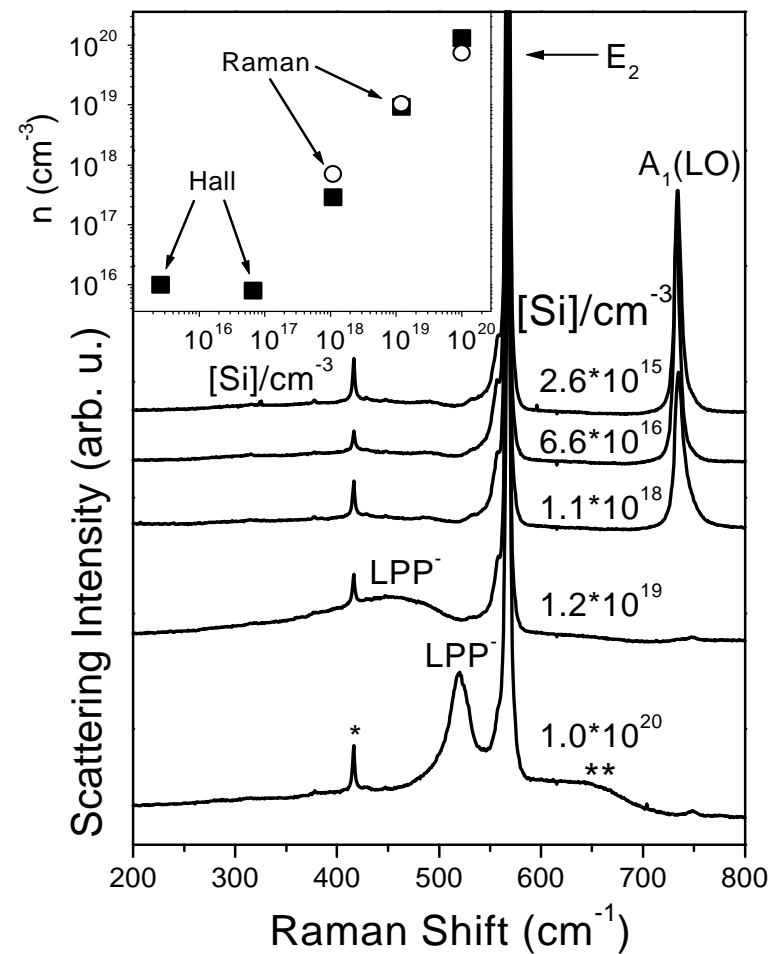

Figure 5: Room-temperature Raman spectra of different $\mathrm{Si}$-doped samples. The inset shows the carrier concentration determined by Hall and Raman measurements vs. Si-content. and by Raman spectroscopy as a function of the silicon concentration. Both data sets agree well. Raman spectroscopy offers the advantage that the free-carrier concentration can be determined with a spatial resolution better than $1 \mu \mathrm{m}$ without contacting the samples.

In equation (5) $\mathrm{n}$ and $\mathrm{m}^{*}$ are the concentration and effective mass of the charge carriers, $\varepsilon_{\infty}$ the highfrequency dielectric constant.

In Figure 5 the spectra of five samples with silicon concentrations between $2.6 \cdot 10^{15} \mathrm{~cm}^{-3}$ (top) and $1.0 \cdot 10^{20} \mathrm{~cm}^{-3}$ (bottom) are plotted. The two highest doped samples exhibit LPP modes at $450 \mathrm{~cm}^{-1}$ and $520 \mathrm{~cm}^{-1}$. The $\mathrm{A}_{1}(\mathrm{LO})$ mode already vanished and therefore the $\mathrm{LPP}^{+}$mode appears at $2580 \mathrm{~cm}^{-1}$ and $1070 \mathrm{~cm}^{-1}$ (not shown). From equations (4) and (5) we calculated the corresponding carrier concentration to be $7.4 \cdot 10^{19} \mathrm{~cm}^{-3}$ and $1.0 \cdot 10^{19} \mathrm{~cm}^{-3}$, respectively. Notice that in both spectra a structure at around $650 \mathrm{~cm}^{-1}(* *)$ appears which is only known from highly doped $\mathrm{GaN}$ [19]. The $\mathrm{A}_{1}(\mathrm{LO})$ mode of the sample with a silicon concentration of about $1.1 \cdot 10^{18} \mathrm{~cm}^{-3}$ appears slightly asymmetric typical for carrier concentrations between $5 \cdot 10^{17}$ $\mathrm{cm}^{-3}$ and $1 \cdot 10^{18} \mathrm{~cm}^{-3}$. For the remaining two samples the free-carrier concentration is too low to cause any shift or broadening of the $\mathrm{A}_{1}(\mathrm{LO})$ mode and cannot be determined by this method. The inset of Figure 5 compares the free-carrier concentration determined by Hall measurements 


\section{Conclusions}

In this work we have shown that a variety of information about doped GaN crystals can be obtained by Raman spectroscopy. We found LVM of magnesium in the region of the host lattice phonons as well as in the high-energy range which now may serve as an indicator for the incorporation of magnesium. No LVM were observed for our Si- and C-doped $\mathrm{GaN}$ samples. Doping with atoms of a different size than the substituted host atom causes internal stress which can be obtained via the shift of the nonpolar $\mathrm{E}_{2}$ mode. For $\mathrm{Mg}$-doped samples we found that the biaxial stress increases with dopant concentration while in case of silicon the size effect is roughly compensated by the increasing free-carrier concentration. The free carrier concentration of doped GaN can be determined by the frequency of the LPP modes.

\section{$\underline{\text { Acknowledgment }}$}

We thank W. Kriegseis (University of Gießen) and M. Straßburg (TU Berlin) for carrying out the SIMS measurements. A.K. acknowledges the support of an Ernst von Siemens scholarship and H.S. a DAAD fellowship.

\section{$\underline{\text { References }}$}

[1] See for example, S. N. Mohammad, A. Salvador, and H. Morkoç, Proc. of the IEEE 83, 1306 (1995).

[2] C. Kisielowski, J. Krüger, S. Ruvimov, T. Suski, J. W. Ager III, E. Jones, Z. Lilienthal- Weber, M. Rubin, E. R. Weber, M. D. Bremser, and R. F. Davis, Phys. Rev. B 54, 17745 (1996).

[3] P. Perlin, J. Camassel, W. Knap, T. Taliercio, J. C. Chervin, T. Suski, I. Grzegory, S. Porowski, Appl. Phys. Lett. 67, 2524 (1995).

[4] A. S. Barker, Jr. and A. J. Sievers, Rev. Mod. Phys. 47, Suppl. 2, S1 (1975).

[5] M. S. Brandt, J. W. Ager III, W. Götz, N. M. Johnson, J. S. Harris, R. J. Molnar, and T. D. Moustakas, Phys. Rev. B 49, R14758, (1994).

[6] W. Götz, N. M. Johnson, D. P. Bour, M. D. McCluskey, and E. E. Haller, Appl. Phys. Lett. 69, 3725 (1996).

[7] S. Einfeldt, U. Birkle, C. Thomas, M. Fehrer, H. Heinke, and D. Hommel, Mat. Sci. Eng. B 50, 12 (1997).

[8] G.-C. Yi and B. W. Wessels, Appl. Phys. Lett. 70, 357 (1997).

[9] J. Neugebauer and C. G. van de Walle, Phys. Rev. Lett. 75, 4452 (1995).

[10] C. G. van de Walle, Phys. Rev. B 56, R10020 (1997).

[11] A. Kaschner, H. Siegle, M. Straßburg, A. Hoffmann, C. Thomsen, U. Birkle, S. Einfeldt, and D. Hommel: "Lowenergy lattice vibrations in Mg-doped GaN grown by MBE", submitted to Appl. Phys. Lett.

[12] W. Limmer, W. Ritter, R. Sauer, B. Mensching, C. Liu, and B. Rauschenbach, Appl. Phys. Lett. 72 , 2589 (1998).

[13] H. Siegle, G. Kaczmarczyk, L. Filippidis, A. P. Litvinchuk, A. Hoffmann, and C. Thomsen, Phys. Rev. B 55, 7000 (1997).

[14] J. C. Nipko, C.-K. Loong, C. M. Balkas and R. F. Davis, Appl. Phys. Lett. 73, 34 (1998).

[15] M. Kuball, F. Demangeot, J. Frandon, M. A. Renucci, J. Massies, N. Grandjean, R. L. Aulombard, and O. Briot, Appl. Phys. Lett. 73, 960 (1998).

[16] T. Kozawa, T. Kachi, H. Kano, H. Nagase, N. Koide, and K. Manabe, J. Appl. Phys. 77, 4389 (1995).

[17] I.-H. Lee, I.-H. Choi, C.-R. Lee, E.-J. Shin, D. Kim, S. K. Noh, S.-J. Son, K. Y. Lim, and H. J. Lee, J. Appl. Phys. 83, 5787 (1998).

[18] M. Leszczynski, H. Teisseyre, T. Suski, I. Grzegory, M. Bockowski, J. Jun, S. Porowski, K. Pakula, J. M. Baranowski, C. T. Foxon, T. S. Cheng, Appl. Phys. Lett. 69, 73 (1996).

[19] F. Demangeot, J. Frandon, M. A. Renucci, C. Meny, O. Briot, and R. L. Aloumbard, J. Appl. Phys. 82, 1305 (1997). 